\title{
The yfhQ gene of Escherichia coli encodes a tRNA:Cm32/Um32 methyltransferase
}

\author{
Elzbieta Purta1,2, Françoise van Vliet ${ }^{3}$, Karolina L Tkaczuk ${ }^{1,4}$, \\ Stanislaw Dunin-Horkawicz ${ }^{1}$, Hirotada Mori ${ }^{5}$, Louis Droogmans ${ }^{6}$ and \\ Janusz M Bujnicki*1,7
}

\begin{abstract}
Address: ${ }^{1}$ Laboratory of Bioinformatics and Protein Engineering, International Institute of Molecular and Cell Biology, ul. ks. Trojdena 4, 02-109 Warsaw, Poland, 2Institute of Biochemistry and Biophysics PAS, Pawinskiego 5a, 02-106 Warsaw, Poland, ${ }^{3}$ Institut de Recherches Microbiologiques Wiame, avenue E. Gryson 1, B-1070 Bruxelles, Belgium, ${ }^{4}$ Institute of Technical Biochemistry, Technical University of Lodz, B. Stefanowskiego 4/10, 90-924 Lodz, Poland, 5 Institute of Advanced Biosciences, Keio University, Tsuruoka, Yamagata 997-0035, Japan, ${ }^{6}$ Laboratoire de Microbiologie, Université Libre de Bruxelles, Institut de Recherches Microbiologiques Wiame, avenue E. Gryson 1, B-1070 Bruxelles, Belgium and ${ }^{7}$ Institute of Molecular Biology and Biotechnology, Adam Mickiewicz University, Umultowska 89, 61-614 Poznan, Poland

Email: Elzbieta Purta - ela@genesilico.pl; Françoise van Vliet - fvvliet@ulb.ac.be; Karolina L Tkaczuk - poison-ivy@genesilico.pl; Stanislaw Dunin-Horkawicz - sdh@genesilico.pl; Hirotada Mori - hmori@gtc.naist.jp; Louis Droogmans - ldroogma@ulb.ac.be; Janusz M Bujnicki* - iamb@genesilico.pl

* Corresponding author
\end{abstract}

Published: 18 July 2006

BMC Molecular Biology 2006, 7:23 doi:10.1186/147|-2199-7-23
Received: II January 2006

Accepted: 18 July 2006

This article is available from: http://www.biomedcentral.com/147I-2199/7/23

(c) 2006 Purta et al; licensee BioMed Central Ltd.

This is an Open Access article distributed under the terms of the Creative Commons Attribution License (http://creativecommons.org/licenses/by/2.0), which permits unrestricted use, distribution, and reproduction in any medium, provided the original work is properly cited.

\begin{abstract}
Background: Naturally occurring tRNAs contain numerous modified nucleosides. They are formed by enzymatic modification of the primary transcripts during the complex RNA maturation process. In model organisms Escherichia coli and Saccharomyces cerevisiae most enzymes involved in this process have been identified. Interestingly, it was found that tRNA methylation, one of the most common modifications, can be introduced by S-adenosyl-L-methionine (AdoMet)-dependent methyltransferases (MTases) that belong to two structurally and phylogenetically unrelated protein superfamilies: RFM and SPOUT.

Results: As a part of a large-scale project aiming at characterization of a complete set of RNA modification enzymes of model organisms, we have studied the Escherichia coli proteins YibK, LasT, YfhQ, and YbeA for their ability to introduce the last unassigned methylations of ribose at positions 32 and 34 of the tRNA anticodon loop. We found that YfhQ catalyzes the AdoMet-dependent formation of $\mathrm{Cm} 32$ or $\mathrm{Um} 32$ in tRNA ${ }^{\text {Serl }}$ and tRNA $\mathrm{Aln}^{\mathrm{In}}$ and that an $E$. coli strain with a disrupted yfhQ gene lacks the tRNA:Cm32/Um32 methyltransferase activity. Thus, we propose to rename YfhQ as $\operatorname{TrMet}(\mathrm{Xm32})$ according to the recently proposed, uniform nomenclature for all RNA modification enzymes, or TrmJ, according to the traditional nomenclature for bacterial tRNA MTases.

Conclusion: Our results reveal that methylation at position 32 is carried out by completely unrelated $\operatorname{TrMet}\left(\mathrm{Xm}_{32}\right)$ enzymes in eukaryota and prokaryota (RFM superfamily member Trm7 and SPOUT superfamily member TrmJ, respectively), mirroring the scenario observed in the case of the $\mathrm{m}^{\prime} \mathrm{G} 37$ modification (introduced by the RFM member Trm5 in eukaryota and archaea, and by the SPOUT member TrmD in bacteria).
\end{abstract}




\section{Background}

All mature transfer RNAs (tRNA) molecules, from all known living organisms, contain numerous modified nucleosides at multiple positions [1]. Although other RNA species also contain modified nucleosides, they are less common than in tRNA, where over 80 modifications have been found to occur at typically $~ 10 \%$, but sometimes even at as many as $25 \%$ positions [2]. Recently, owing to the availability of complete genome sequences, there has been a remarkable progress in identification of enzymes that introduce modifications in tRNAs of model organisms Escherichia coli and Saccharomyces cerevisiae (reviews: $[3,4]$ ). For instance, as a part of a large-scale project, aiming at identification of the complete repertoire of RNA methyltransferases (MTases) in E. coli by combination of bioinformatics and experimental analyses, we have recently identified the so far "missing" enzymes that introduce $\mathrm{m}^{7} \mathrm{G} 46$ [5] and $\mathrm{mnm}^{5} \mathrm{~s}^{2} \mathrm{U} 34$ [6]. In E. coli the only tRNA methylations, for which the corresponding enzymes (and genes that encode them) remain unknown, are those of the 2'-OH groups of ribose at positions 32 and 34 of the anticodon loop (Figure 1).

Ribose methylation is one of the most common modifications. In E. coli it was found to be introduced at different positions of different RNAs by site-specific enzymes, $S$ adenosyl-L-methionine (AdoMet)-dependent MTases that belong to two structurally and phylogenetically unrelated protein superfamilies: Rossmann-fold MTase (RFM) [7,8] and SPOUT [9]. These superfamilies have been defined based on sequence and structural comparisons (review: [10]). The RFM superfamily can be exemplified by the $23 S$ rRNA:Um2552 MTase RrmJ (which is also able to methylate tRNA in vitro, albeit at an unspecified position) $[11,12]$, while the SPOUT superfamily can be exemplified by the tRNA:Gm18 MTase TrmH $[13,14]$.

Previously, we found that in $S$. cerevisiae methylations at positions 32 and 34 are introduced by the Trm7 enzyme, a close homolog of RrmJ and a member of the RFM superfamily [15]. However, apart from the rRNA MTase RrmJ, there are no close homologs of Trm7 in E. coli that could carry out the corresponding reactions in tRNAs [16], which suggests that modifications at positions 32 and/or 34 in prokaryotic and eukaryotic tRNAs could be carried out by analogous, i.e. unrelated proteins. To test this hypothesis, we assayed the so far functionally uncharacterized members of the SPOUT superfamily [9]: YibK, LasT, YfhQ, and YbeA for their ability to exert methylation of tRNA at positions 32 and/or 34 .

\section{Results and discussion \\ Preparation of the substrates and methyltransferase candidates}

Analysis of the tRNA sequences and modified nucleosides in the MODOMICS database [17] revealed the presence of 2'-O-methylated uridine (Um) in position 32 of the anticodon loop in tRNAGln1 (UUG) and tRNAGln2 (CUG), and 2'-O-methylated cytidine $(\mathrm{Cm})$ at the same position in $\mathrm{tRNA}_{\mathrm{f}}$ Met1 (CAU), tRNA $\mathrm{fet}^{\mathrm{Me}}$ (CAU), tRNA ${ }^{\mathrm{Ser} 1}$ (UGA), and tRNA $^{\operatorname{Trp} 1}$ (CCA). On the other hand, the nucleoside in position 34 was found to be $2^{\prime}$-O-methylated in tRNALeu4 (UAA) to 5-carboxymethylaminomethyl-2-O-methyluridine $\left(\mathrm{cmnm}^{5} \mathrm{Um}\right)$, and in tRNA ${ }^{\text {Leu5 }}$ (CAA) to $\mathrm{Cm}$. We selected tRNA ${ }^{\text {Ser } 1}$ and tRNA ${ }^{\text {Gln}} 2$ as representative substrates for the methylation at position 32, while tRNALeu5 was selected as a representative substrate for the methylation at position 34. Transcripts were generated in vitro using T7-RNA polymerase (see Methods).

Among the cloned open reading frames without experimentally assigned functions, we selected a set of putative MTases identified as candidates for RNA modification enzymes in earlier studies: YibK, LasT, YfhQ, and YbeA $[9,18]$. All these proteins are members of the SPOUT superfamily of MTases [9], which is characterized by the presence of a deep topological knot $[19,20]$. The experimental analysis of these putative tRNA modification enzymes in E. coli was greatly facilitated by the availability of a complete set of cloned individual genes encoding Histagged proteins [21] and the corresponding knock-out (K.O.) strains [22], which should lack the corresponding MTase activities and hence, contain unmethylated substrates. Thus, on the one hand, total tRNA was extracted from the $y i b K, l a s T, y f h Q$, and $y b e A$ K.O. strains. On the other hand, we have expressed and purified His-tagged YibK, LasT, YfhQ, and YbeA proteins. Here, data is shown in detail only for YfhQ, as it was the only MTase candidate, for which we were able to demonstrate the tRNA MTase activity using the selected substrates. SDS-PAGE analysis of the fractions containing YfhQ in the presence of $7.5 \% \quad \beta$-mercaptoethanol showed one band corresponding to approximately $30 \mathrm{kDa}$ (Figure 2), in agreement with the theoretical value calculated for the YfhQ monomer (29.168 kDa). The immunoblot analysis using anti-His tag antibodies confirmed the presence of a Histagged protein in the $30 \mathrm{kDa}$ band (data not shown). Interestingly, SDS-PAGE analysis in the lower concentration of $\beta$-mercaptoethanol (e.g. 5\%) revealed an additional band of approximately $60 \mathrm{kDa}$, suggesting the existence of a very strong dimer. It was verified by Western blot analysis as containing the His6 protein (data not shown), this suggesting that it is indeed the dimeric form of the $30 \mathrm{kDa}$ protein. Gel filtration chromatography revealed that the apparent molecular mass of the native form of the YfhQ protein is about $58 \mathrm{kDa}$ (Figure 3). Thus, 


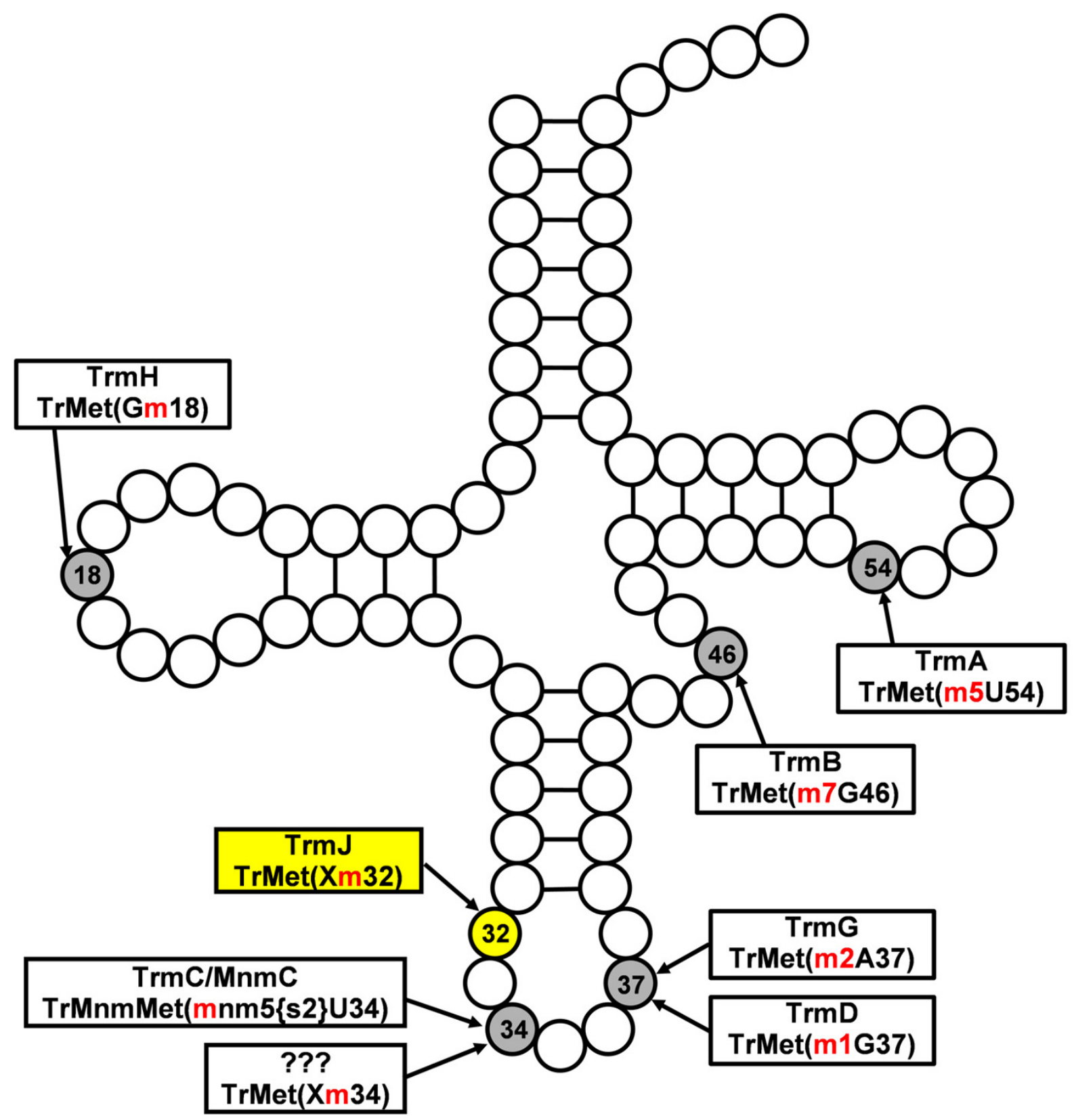

Figure I

The cloverleaf representation of tRNAs from E. coli, with known methylated nucleosides and the respective enzymes indicated.

we conclude that YfhQ exists as a strong dimer, similarly to its homologs from the SPOUT superfamily with known structures (e.g. YibK [23]). The results of SDS-PAGE and gel filtration analyses for YibK, LasT, and YbeA were qualitatively similar, e.g. their molecular mass agreed with the theoretical values expected for monomers and dimers (data not shown).
yfhQ encodes a MTase responsible for the formation of Cm/Um 32 in the anticodon loop of tRNA

In order to test whether YfhQ, YibK, LasT, or YbeA could methylate the $\mathrm{U}$ or $\mathrm{C}$ nucleosides in positions 32 or $\mathrm{C}$ in position 34 of the anticodon loop, we incubated the purified proteins with AdoMet and with in vitro transcribed tRNAs: [ $\alpha$-32P]CTP-labelled tRNASer1, [ $\alpha$-32P]UTP-labelled 


\section{6}

\section{6,2}

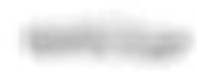

45

35

25
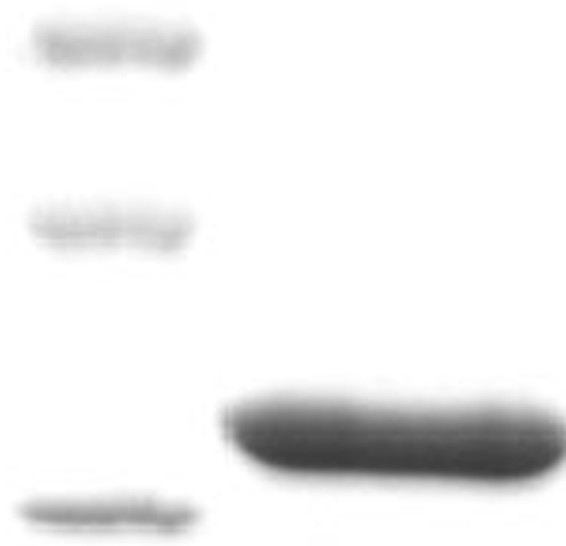

18,4

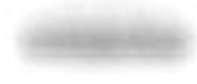

\section{4,4}

\section{Figure 2}

SDS-PAGE analysis of the purified $Y$ fhQ protein. Left lane, molecular mass markers (Pharmacia-Biotech). Right lane, purified recombinant YfhQ.

tRNAGln2, and [ $\alpha^{32}$ P]CTP-labelled tRNALeu5. Combinations of every protein with every tRNA substrate were tested. After incubation, the tRNA was hydrolyzed using nuclease P1 and the resulting 5'-phosphate nucleosides were analyzed by two-dimensional cellulose thin layer chromatography (2D-TLC) followed by autoradiography. The results shown in Figure 4 demonstrate that YfhQ is able to introduce $\mathrm{Cm}$ in the in vitro transcribed tRNA ${ }^{\mathrm{Ser} 1}$ and Um in the in vitro transcribed tRNAGln2. We were unable to detect the MTase activity of YibK, LasT, or YbeA on any of these substrates (data not shown), therefore they were not analyzed further.

In order to further confirm that YfhQ is responsible for methylation at position 32, a similar experiment was performed using $[\alpha-32 P] U T P-l a b e l l e d$ tRNA ${ }^{\text {Ser1 }}$. After incubation in the presence of AdoMet and purified YfhQ, the tRNA was hydrolyzed by ribonuclease (RNase) T2, which cleaves the phosphodiester bonds to generate 3'-monophosphate nucleosides but does not cut the phosphodiester bond between a 2'-O-methylated nucleoside and the 3'-adjacent nucleoside, leaving 2'-O-methylated dinucleotides. The analysis of cleavage products revealed the presence of radioactively labelled CmUp (Figure 5), demonstrating that YfhQ methylates a $\mathrm{C}$ nucleoside $5^{\prime}$ adjacent to a U. A similar experiment performed using [ $\alpha$ ${ }^{32} \mathrm{P}$ ]UTP-labelled tRNAGln2 revealed the formation of radioactively labelled UmUp (Figure 5). These results are perfectly consistent with the expected 2'-O-methylation at position 32 (followed by $U$ both in tRNASer1 and tRNAGln2).

We confirmed the localization of the 2'-O-methyl group by an independent approach. [ $\alpha-32$ P]UTP-labelled tRNASer1 was modified using purified YfhQ and then cleaved by RNAse T1. The resulting oligonucleotides $(8,6$, 5 and 4-nt long fragments) were separated on a $20 \%$ polyacrylamide gel and revealed by autoradiography. The oligonucleotides were eluted from gel and cleaved with RNAse T2. The TLC analysis of cleavage products revealed the presence of radioactively labelled CmUp (Figure 6) derived from a 5-nt fragment, demonstrating that YfhQ methylates a $\mathrm{C}$ nucleoside 5 '-adjacent to a U nucleoside in a given nucleotide context, encompassing position 32 .

To determine whether the Cm32/Um32 MTase activity was affected in the $y f h Q$ K.O. strain, cell extracts of $E$. coli MC1061 and of $y f h Q$ K.O. strains were incubated with AdoMet and $\left[\alpha-{ }^{32} \mathrm{P}\right] \mathrm{CTP}$-labelled in vitro transcribed tRNA ${ }^{\text {Ser } 1}$ or $[\alpha-32 \mathrm{P}] \mathrm{UTP}$-labelled tRNAGln2. After incubation, tRNA was hydrolyzed by nuclease P1 and the nucleotides were analyzed by 2D-TLC and autoradiography. The results shown in Figure 7 revealed the absence of $\mathrm{Cm} /$ Um formation in $y f h Q$ K.O. extract. This result was complemented by transforming the $y f h Q$ K.O. strain with a plasmid carrying the $y f h Q$ gene, purifying the YfhQ protein from that strain, as described in the Methods section, and testing it for the MTase activity (Figure 8). The observed activity allows us to conclude that YfhQ is essential for the Cm32/Um32 methylation. 


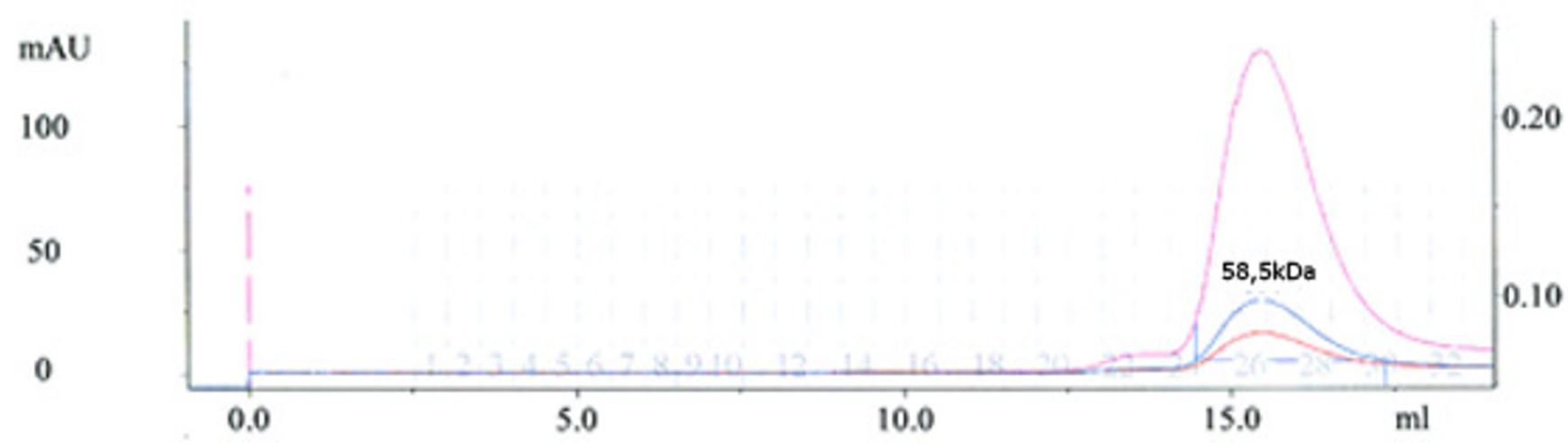

Figure 3

Gel-filtration profile of the purified YfhQ protein. Magenta line: UV $232 \mathrm{~nm}$, red: UV $260 \mathrm{~nm}$, blue: UV $280 \mathrm{~nm}$. The peak at $16 \mathrm{ml}$ corresponds to a dimeric form of YfhQ.

We also found that total (crude) tRNA extracted from the wild-type strain MC1061 was not a substrate for the purified YfhQ enzyme, while tRNA from the $y f h Q$ K.O. strain was an excellent substrate for this enzyme. The purified YfhQ protein was incubated with ${ }^{14} \mathrm{C}$-radiolabelled AdoMet ([methyl-14C]AdoMet) and total tRNA extracted either from the wild type strain MC1061 (supposedly fully methylated) or from the yfhQ_K.O. strain (supposedly unmethylated in the position specific for the YfhQ MTase). After incubation, the tRNA was hydrolyzed by nuclease P1 and the resulting nucleotides were analyzed by 2D-TLC and autoradiography. As expected, the result shown in Figure 9 revealed the formation of a radioactive compound with migration characteristic for $\mathrm{Cm}$ only in the case of tRNA from the $y f h Q$ K.O. strain, but not in the case of the fully modified tRNA. This suggests that in E. coli YfhQ is the only MTase responsible for the formation of Cm32 in tRNA. Curiously, no Um was detected in this last experiment. The cause could be that tRNAs that normally contain Um32 are much less abundant than tRNAs containing $\mathrm{Cm} 32$. However, the possibility that another MTase can also form Um32 cannot be totally excluded. In accordance with the recently proposed, uniform nomenclature for RNA modification enzymes [17] we suggest to rename YfhQ as TrMet(Xm32). Alternatively, according to the traditional nomenclature for bacterial tRNA MTases, it could be named TrmJ.

\section{Sequence analysis and modeling of YfhQ reveals a} conserved active site common to ribose 2'-O-MTases from the SPOUT superfamily and underscores the convergent evolution with the RFM superfamily

YfhQ was previously reported to belong to the SPOUT superfamily of MTases [9], although at that time no structural information was available for any of these proteins to guide the identification of the active site. Only recently, a number of SPOUT structures were solved, providing templates for homology modeling of other members. We carried out the protein fold-recognition analysis for the YfhQ sequence using the GeneSilico meta-server [24] to predict its structure. We found, as expected, that the structures of SPOUT superfamily members were identified as the only compatible templates for $\mathrm{YfhQ}$, with genuine ribose MTases RlmB [19], TrmH [14], and AviRb [25], as well as putative MTases RrmA [20] and YibK [23] reported with highest scores (Figure 10). We constructed a homology model of YfhQ using the "FRankenstein Monster's approach" [26,27]. Comparison of the model with the templates (Figure 11) reveals the common "knotted" structure of the AdoMet-binding site and conservation of the residues demonstrated to be involved in catalysis in TrmH [28]. This suggests that tRNA MTases TrmH and YfhQ use a very similar mechanism for methylation of ribose in different positions, 18 and 32, respectively. The same active site is also conserved in YibK, which we found not to methylate tRNA and which we predict to be involved in modification of one (or more) of the "unassigned" ribose methylations in rRNA [29]. We predict that the tRNA-binding activity and substrate specificity of YfhQ is at least in part dependent on the presence of the C-terminal extension, which is absent from other members of this family (e.g. no extensions in YibK) or replaced by different domains or extensions implicated in RNAbinding (e.g. additional helices at $\mathrm{N}$ - and C-termini present in TrmH or the N-terminal domain in RlmB and AviRb).

The statistical significance of sequence conservation between YfhQ and previously characterized SPOUT MTases of known structure (expectation value $3^{*} 10^{-16}$ in the $2^{\text {nd }}$ iteration of PSI-BLAST [30]) practically guarantees that their structures are very similar. Experimental support for this prediction is obtained from the Circular Dichroim (CD) analysis (Figure 12, see Methods for details), which 
a
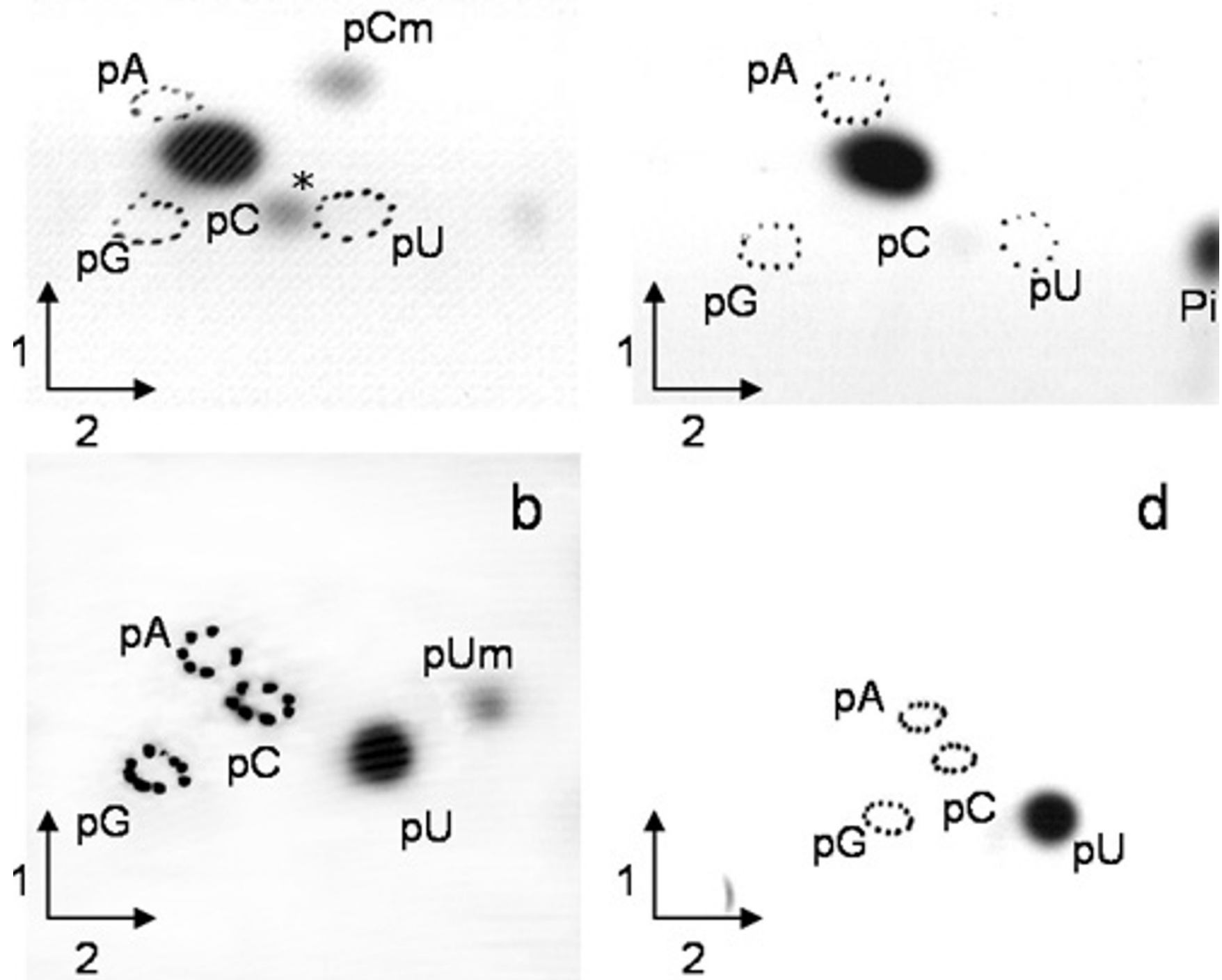

Figure 4

Nuclease PI cleavage of in vitro transcribed E. coli tRNASerI and tRNA ${ }^{\text {Gln2 }}$ incubated with YfhQ and AdoMet. Autoradiography of two-dimensional chromatograms of 5'-phosphate and 3'-phosphate nucleosides on thin layer cellulose plates. [ $\alpha-32$ P]CTP-labelled in vitro transcribed tRNA ${ }^{\text {Serl }}\left(2.5 \times 10^{5} \mathrm{cpm}\right)(\mathrm{a}$ and $\mathrm{c})$ and $[\alpha-32 \mathrm{P}] \mathrm{CTP}$-labelled in vitro transcribed tRNA $\operatorname{Gin}^{2}\left(2.5 \times 10^{5} \mathrm{cpm}\right)(\mathrm{b}$ and d) was incubated in the presence $(\mathrm{a}$ and $\mathrm{b})$ or absence $(\mathrm{c}$ and $\mathrm{d})$ of the YfhQ protein. The reaction mixture contained $50 \mathrm{mM}$ PIPES-Na, pH 7.0, $4 \mathrm{mM} \mathrm{MgCl}, 50 \mu \mathrm{M}$ AdoMet and $5 \mu \mathrm{g}$ of the purified YfhQ protein. After 90 min incubation at $37^{\circ} \mathrm{C}$, the tRNA was recovered, cleaved by nuclease $\mathrm{PI}$, the resulting nucleotides were analyzed as described [34]. The additional spot marked with an asterisk ("*") corresponds to an unknown species, a product either of an unusual cleavage pattern modified by the presence of 2'-O-methylation or degradation. 

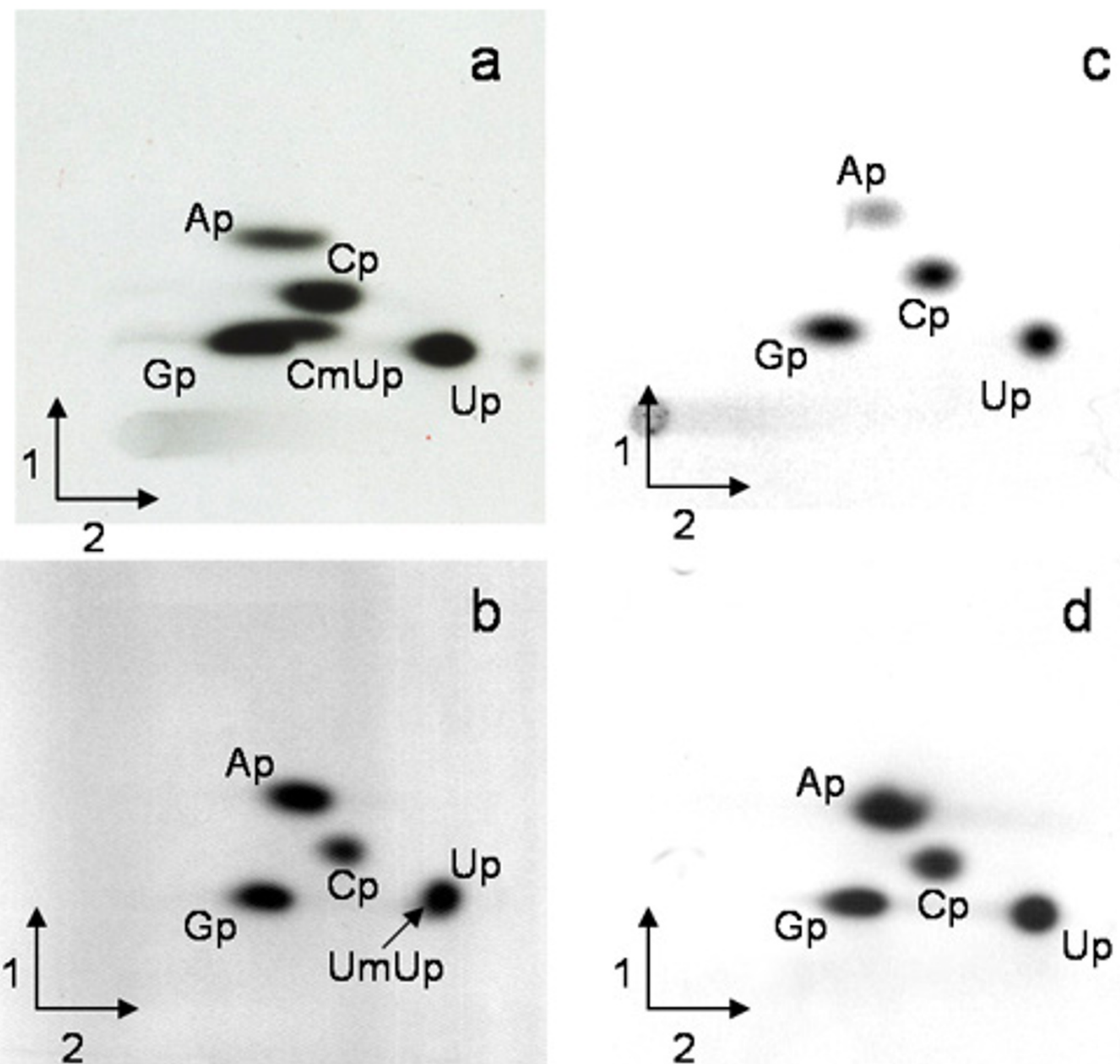

\section{Figure 5}

RNAse T2 cleavage of in vitro transcribed E. coli tRNASerI and tRNAGln2 incubated with YfhQ and AdoMet. Autoradiography of two-dimensional chromatograms of 5'-phosphate and 3'-phosphate nucleosides on thin layer cellulose plates. [ $\alpha-{ }^{32 P}$ ]UTP-labelled in vitro transcribed tRNA ${ }^{\text {Serl }}\left(2.5 \times 10^{5} \mathrm{cpm}\right)(\mathrm{a}$ and $\mathrm{c})$ and $\left[\alpha-{ }^{32} \mathrm{P}\right] \mathrm{UTP}$-labelled in vitro transcribed tRNAGln2 $\left(2.5 \times 10^{5} \mathrm{cpm}\right)(\mathrm{b}$ and $d)$ was incubated in the presence $(\mathrm{a}$ and $\mathrm{b})$ or absence $(\mathrm{c}$ and $d)$ of the YfhQ protein. The reaction mixture contained $50 \mathrm{mM}$ PIPES-Na, pH 7.0, $4 \mathrm{mM} \mathrm{MgCl}, 50 \mu \mathrm{M}$ AdoMet and $5 \mu \mathrm{g}$ of the purified YfhQ protein. After 90 min incubation at $37^{\circ} \mathrm{C}$, the tRNA was recovered, cleaved by RNAse $\mathrm{T} 2$, the resulting nucleotides were analyzed as described [38]. 


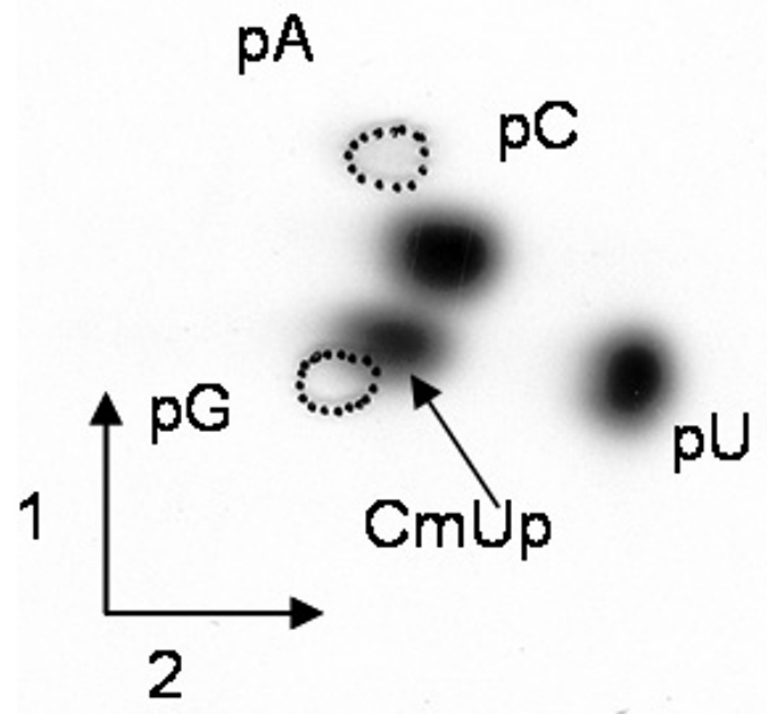

\section{Figure 6}

Complete RNAse TI cleavage followed by nuclease T2 cleavage of in vitro transcribed $E$. coli tRNASer I incubated with YfhQ and AdoMet. Autoradiography of a two-dimensional chromatogram of 5'-phosphate nucleosides on a thin layer cellulose plate. $[\alpha-32 \mathrm{P}] \mathrm{CTP}$-labelled in vitro transcribed tRNASerl $\left(1.5 \times 10^{6} \mathrm{cpm}\right)$ was incubated with the YfhQ protein. The reaction mixture contained $50 \mathrm{mM}$ PIPES$\mathrm{Na}, \mathrm{pH} 7.0,4 \mathrm{mM} \mathrm{MgCl}, 50 \mu \mathrm{M}$ AdoMet and $5 \mu \mathrm{g}$ of the purified $\mathrm{YfhQ}$ protein. After $90 \mathrm{~min}$ incubation at $37^{\circ} \mathrm{C}$, the tRNA was recovered, digested overnight at $37^{\circ} \mathrm{C}$ by $400 \mathrm{u}$ RNAse $\mathrm{TI}$ in $100 \mathrm{mM}$ Tris- $\mathrm{HCl} \mathrm{pH}$ 7.5. The resulting oligonucleotides were separated on a $20 \%$ polyacrylamide gel (19:I) and revealed by autoradiography. Oligonucleotides were eluted from the gel and cleaved by nuclease T2. The resulting nucleotides were analyzed as described [38]. This image shows the TLC analysis for a 5-nucleotide long fragment (nucleotides 3I-35).

reveals that the secondary structure content of YfhQ (approximately $43 \%$ of helices and $23 \%$ of strands) is similar to the prediction reported here, taking into account the helical structure of the unmodeled C-terminal region. These values are also comparable to the secondary structure observed in the crystal structure of a bona fide SPOUT member YibK and our measurements of CD spectra carried out for YibK (data not shown).

\section{Conclusion}

Our results reveal that methylation at position 32 in the anticodon loop of bacterial and eukaryotic tRNAs is carried out by completely unrelated enzymes: a SPOUT superfamily member YfhQ and RFM superfamily member Trm7. This scenario is strikingly similar to the one observed in the case of $\mathrm{m}^{1} \mathrm{G} 37$ modification, which is carried out by the SPOUT superfamily member TrmD in bacteria and by the RFM superfamily member Trm 5 in eukaryota and archaea $[31,32]$. On the other hand, methylation of ribose at position 18 is carried out by members of the SPOUT superfamily both in prokaryota and eukaryota: $\operatorname{TrmH}$ [13], and $\operatorname{Trm} 3$ [33], respectively, while the $N^{1}$. methylation of adenosine A58 (not observed in E. coli, but e.g. in Thermus thermophilus) is catalyzed by members of the RFM superfamily [34,35]. It is unclear which of these two MTase superfamilies was more ancient and how they replaced each other for the methylation of different positions in tRNAs from different phylogenetic lineages. Among the four members of the SPOUT superfamily studied in this work (YfhQ, YibK, LasT, and YbeA) we were unable to identify the MTase specific for the position 34 in E. coli tRNAs. It remains to be determined if this last missing tRNA MTase is present among the remaining, so far uncharacterized members of SPOUT and RFM superfamilies $[8,9]$ and whether it uses a known active site or invented a new one for the same reaction. Definitely, more work is needed to elucidate the complicated pathways of evolution of RNA modification systems in all Domains of Life.

\section{Methods \\ Preparation of the substrates}

Plasmids containing the $y f h Q, y i b K, l a s T$, and $y b e A$ genes inserted into the pCA24N vector and the corresponding $E$. coli K.O. strains were constructed as described earlier $[21,22]$. pBlueScript II KS (+) (Stratagene) has been modified by site-directed mutagenesis to introduce BpiI and Mph1103I cloning sites in pBlueScript II KS (+) polylinker, resulting in pKS_RNA vector. The serT and leuZ genes, encoding tRNA ${ }^{\text {Ser1 }}$ and tRNA ${ }^{\text {Leu5 }}$ respectively, were PCR-amplified from the E. coli genomic DNA using primers 5'-GCATGCATTGGCGGAAGCGCAGAGATTCGAAC$3^{\prime}$ and 5'-GAAGACCCTATAGG AAGTGTGGCCGAGCGGTTG-3' for serT and 5'-TAATGCATGGTACCCGGAG CGGGAC-3' and 5'-AGACCCTATAGCCCGGATGGTGGAATCGGTAG-3' for leuZ. The final PCR product was cloned into the pKS_RNA vector, generating plasmids pKS_SerT and pKS_LeuZ. Transcripts were generated in vitro using T7-RNA polymerase and Mph1103I-cleaved pKS_SerT or pKS_LeuZ plasmids as templates. The tRNAGln2 transcript was generated exactly as described in [36]. Full-length transcripts were purified by $10 \%$ polyacrylamide gel electrophoresis.

\section{Expression and purification of the YfhQ, YibK, LasT, and YbeA recombinant proteins}

Proteins were expressed in E. coli strain BL21 (DE3). Transformed cells were grown at $37^{\circ} \mathrm{C}$ in Luria broth (supplemented with chloramphenicol at $30 \mu \mathrm{g} / \mathrm{mL}$ ) to an optical density at $660 \mathrm{~nm}\left(\mathrm{OD}_{660}\right)$ of 0.7 . At this stage, 


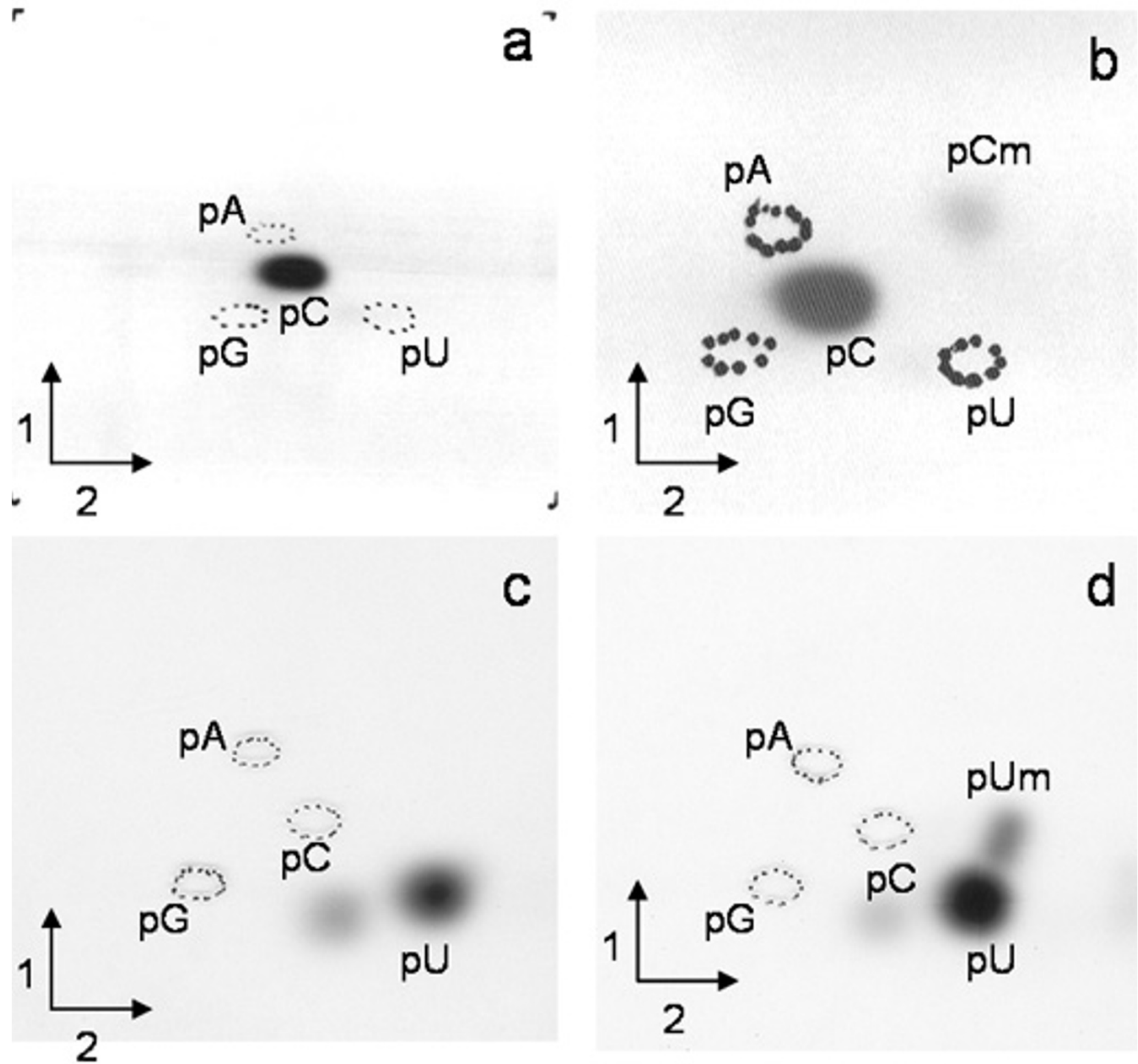

\section{Figure 7}

Nuclease PI cleavage of in vitro transcribed E. coli tRNA ${ }^{\text {Serl }}$ and tRNA ${ }^{\text {Gln2 }}$ incubated with cellular extracts and AdoMet. Autoradiography of two-dimensional chromatograms of 5 '-phosphate nucleosides on thin layer cellulose plates. [ $\alpha$ 32P]CTP-labelled in vitro transcribed tRNA ${ }^{\text {Serl }}\left(2.5 \times 10^{5} \mathrm{cPm}\right)(\mathrm{a}, \mathrm{b})$ and $[\alpha-32 \mathrm{P}] \mathrm{UTP}$-labelled in vitro transcribed tRNA ${ }^{\mathrm{Gln} 2}(2.5 \times$ $\left.10^{5} \mathrm{cpm}\right)(\mathrm{c}, \mathrm{d})$ was incubated with a crude extract of the YfhQ K.O. strain (a and c), or of the MCI06I E. coli strain (b and d). The reaction mixture contained $50 \mathrm{mM}$ PIPES-Na, pH7.0, $4 \mathrm{mM} \mathrm{MgCl}$, $50 \mu \mathrm{M}$ AdoMet and $100 \mu \mathrm{g}$ of total protein. After 90 min incubation at $37^{\circ} \mathrm{C}$, the tRNA was recovered, digested by nuclease PI and the resulting nucleotides were analyzed as described [38]. 


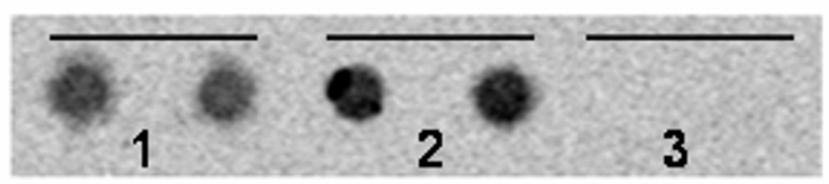

Figure 8

Dot-blot analysis of total tRNA from the yfhQ K.O. strain incubated with $\mathbf{Y} f \mathbf{Q}$ and AdoMet. The reaction mixture containing $50 \mathrm{mM}$ PIPES- $\mathrm{Na}, \mathrm{pH} 7.0,4 \mathrm{mM} \mathrm{MgCl}$, $100 \mu \mathrm{g}$ of total tRNA from $y$ fhQ K.O. strain, $5 \mu \mathrm{g}$ of the purified YfhQ protein (I-YfhQ protein purified from the $y$ fh $Q$ K.O. strain transformed with a plasmid carrying the $y$ fh $Q$ gene, 2 - YfhQ purified from the regular overexpressing strain, 3 - a negative control experiment without any protein), and $10 \mu \mathrm{M}\left[{ }^{14} \mathrm{C}\right.$-methyl] AdoMet $(53 \mathrm{mCi} / \mathrm{mmol})$. After $60 \mathrm{~min}$ incubation at $37^{\circ} \mathrm{C}, 50 \mu$ aliquots were filtered in duplicate in a dot-blot manifold (Scie-Plast) using a DE-8I cellulose filter. The filter was washed three times with $200 \mu \mathrm{l}$ of the reaction buffer and one time with $70 \% \mathrm{EtOH}$, dried and exposed to the Phosphorlmager screen. The resulting image was scanned on the Storm 820 Phosphorlmager.

IPTG (isopropylthiogalactopyranoside) was added up to a final concentration of $1 \mathrm{mM}$ to induce recombinant protein expression. Cells were harvested after 3 hours incubation at $37^{\circ} \mathrm{C}$ and resuspended in buffer A (50 mM Tris$\mathrm{HCl}, \mathrm{pH} 7.5,10 \mathrm{mM} \mathrm{MgCl}_{2}, 10 \%$ glycerol) and lysed by sonication. The lysate was cleared by centrifugation $(20,000 \times g$ during $10 \mathrm{~min})$ and was applied to a column of Chelating Sepharose Fast Flow (Pharmacia Biotech) charged with $\mathrm{Ni}^{2+}$. The column was washed with buffer A supplemented with $5 \mathrm{mM}$ imidazole and the adsorbed material was eluted with a linear gradient $(0.05 \mathrm{M}$ up to $0.4 \mathrm{M}$ ) of imidazole. Eluted fractions were analyzed by SDS-PAGE in the presence of 5-7.5\% $\beta$-mercaptoethanol. In the higher concentration of $\beta$-mercaptoethanol only the monomeric form was observed (Figure 2), while the lower concentrations allowed to observe both the monomeric and dimeric forms. YfhQ was further purified by gel filtration chromatography. The partially purified enzyme was applied on a Superdex 200 column (Pharmacia Biotech) equilibrated with buffer B (50 mM Tris- $\mathrm{HCl}, \mathrm{pH} 7.5$, $150 \mathrm{mM} \mathrm{NaCl}, 10 \mathrm{mM} \mathrm{MgCl}_{2}, 10 \%$ glycerol).

\section{Circular Dichroism analysis}

CD spectra were collected on the Jasco-810 spectropolarimeter with a temperature controller. The concentration of YfhQ protein was $5 \mu \mathrm{M}$ and YibK protein $6.6 \mu \mathrm{M}$. Scans were collected at $20^{\circ} \mathrm{C}$ from 200 to 260 , in $1 \mathrm{~nm}$ steps, using a $1 \mathrm{~mm}$ pathlength cuvette. Secondary structure content was estimated from CD spectrum using the CDpro software [37].

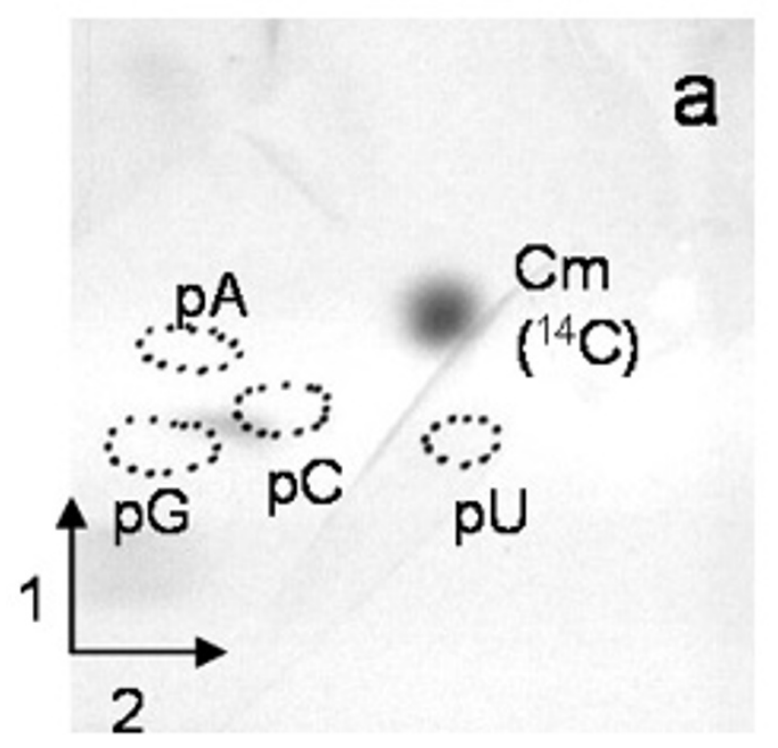

Figure 9

Nuclease PI cleavage of total tRNA from yfhQ K.O. strain incubated with $\mathbf{Y}$ fhQ and AdoMet. Autoradiography of a two-dimensional chromatogram of 5'-phosphate nucleosides on a thin layer cellulose plate. The reaction mixture containing $50 \mathrm{mM}$ PIPES-Na, $\mathrm{pH} 7.0,4 \mathrm{mM} \mathrm{MgCl}, 100$ $\mu \mathrm{g}$ of total tRNA from $y$ fhQ K.O. strain, $5 \mu \mathrm{g}$ of the purified YfhQ protein, and $10 \mu \mathrm{M}\left[{ }^{14} \mathrm{C}\right.$-methyl]AdoMet $(53 \mathrm{mCi} /$ $\mathrm{mmol})$. After $60 \mathrm{~min}$ incubation at $37^{\circ} \mathrm{C}$, the tRNA was recovered, digested by nuclease $\mathrm{PI}$ and the resulting nucleotides were analyzed as described [38].

\section{Authors' contributions}

EP participated in the design, execution and interpretation of all experimental analyses, preparation of the figures and writing of the manuscript. FvV participated in nucleotide analyses by thin layer chromatography. KLT carried out sequence analysis and modeling of the MTase candidates and prepared Figures 9 and 10. SDH participated in tRNA sequence analysis and prepared Figure 1. HM provided constructs expressing YfhQ, YibK, LasT, YbeA and the corresponding knock-out strains. LD coordinated the experimental analyses, and participated in the analysis and interpretation of the data. JMB conceived of the project, coordinated its execution and drafted the manuscript. All authors read and approved the final manuscript.

\section{Acknowledgements}

We thank Catherine Tricot (Institut de Recherches Microbiologiques Wiame, Bruxelles, Belgium) for the help in gel filtration experiments. The work in Poland was supported by the EMBO/HHMI Young Investigator Programme award to J.M.B. The work in Belgium was supported by the Fonds pour la Recherche Fondamentale Collective (FRFC). E.P. was supported by the fellowship from the Commissariat Général aux Relations internationales de la Communauté française de Belgique and by the Centre 


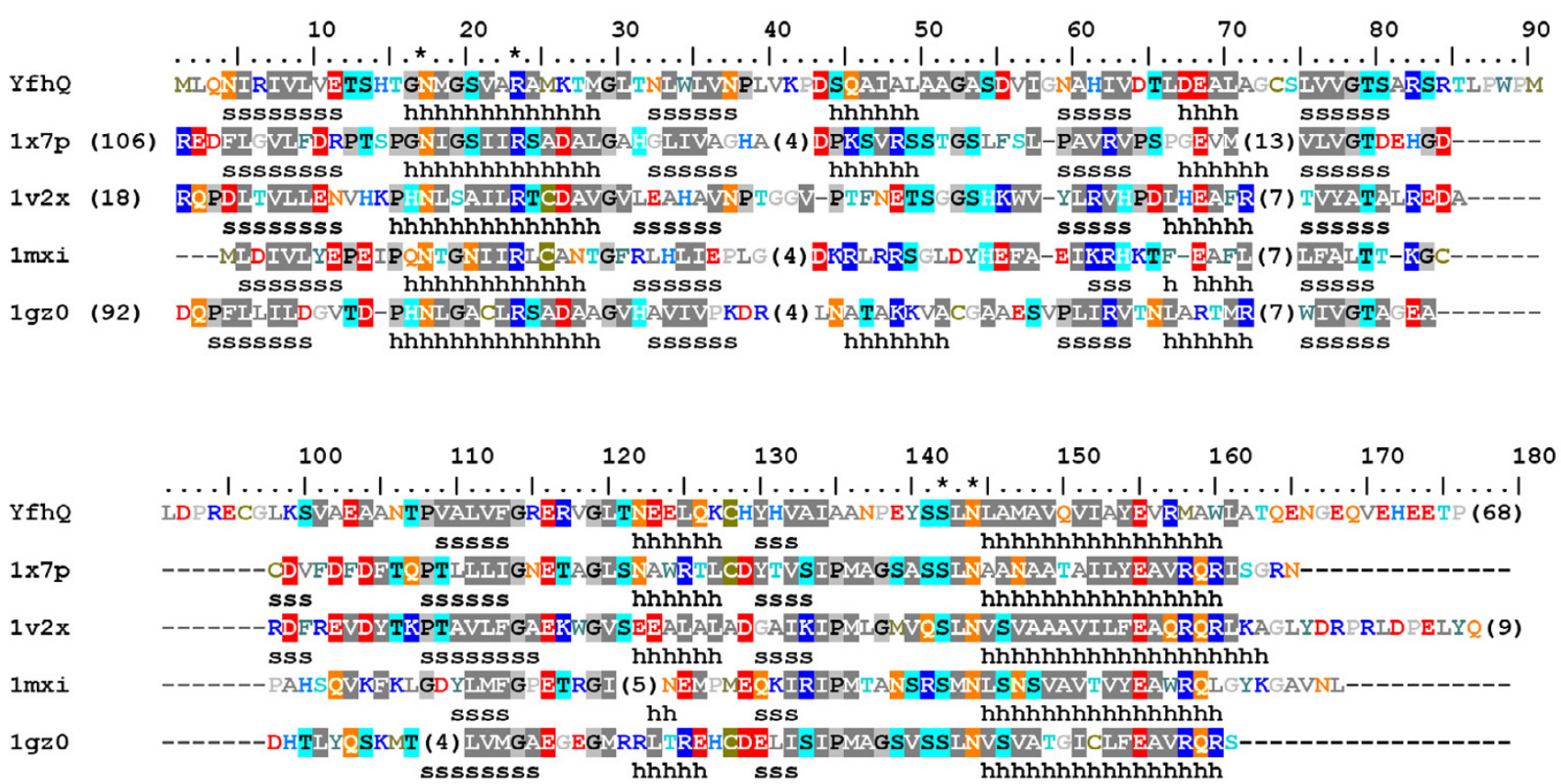

\section{Figure 10}

Fold recognition alignment between YfhQ and the experimentally solved structures of SPOUT MTases. The sequence of $Y$ fhQ has been aligned to the structures of RImB from Escherichia coli (Igz0.pdb), AvirB from Streptomyces viridochromogenes (Ix7p.pdb), TrmH from Thermus thermophilus (Iv2x.pdb), and YibK from Haemophilus influenzae (Imxi.pdb), using the GeneSilico meta server [24]. Amino acid residues predicted to be involved in cofactor binding and in the methyl transfer reaction are indicated by asterisks ("*").

of Excellence in Molecular BioMedicine project within the 5th Framework Programme of the European Commission (contract QLK6-CT-200290363).

\section{References}

I. Bjork GR: Genetic dissection of synthesis and function of modified nucleosides in bacterial transfer RNA. Prog Nucleic Acid Res Mol Biol 1995, 50:263-338.

2. Auffinger $P$, Westhof $E$ : Location and distribution of modified nucleotides in tRNA. In Modification and editing of RNA Edited by: Grosjean H, Benne R. Washington: ASM Press; 1998:569-576.

3. Hopper AK, Phizicky EM: tRNA transfers to the limelight. Genes Dev 2003, I7(2): 162-180.

4. Bujnicki JM, Droogmans L, Grosjean H, Purushothaman SK, Lapeyre $\mathrm{B}$ : Bioinformatics-guided identification and experimental characterization of novel RNA methyltransferases. In Practical Bioinformatics Volume 15. Edited by: Bujnicki JM. Berlin: SpringerVerlag; 2004:139-168.

5. De Bie LG, Roovers M, Oudjama Y, Wattiez R, Tricot C, Stalon V, Droogmans L, Bujnicki JM: The yggH gene of Escherichia coli encodes a tRNA ( $\left.\mathbf{m}^{7} \mathbf{G} 46\right)$ methyltransferase. J Bacteriol 2003, I 85( I 0):3238-3243.

6. Bujnicki JM, Oudjama Y, Roovers M, Owczarek S, Caillet J, Droogmans L: Identification of a bifunctional enzyme MnmC involved in the biosynthesis of a hypermodified uridine in the wobble position of tRNA. Rna 2004, I 0(8): I 236-I 242.

7. Bujnicki JM: Comparison of protein structures reveals monophyletic origin of the AdoMet-dependent methyltransferase family and mechanistic convergence rather than recent differentiation of $\mathbf{N}^{4}$-cytosine and $\mathbf{N}^{6}$-adenine DNA methylation. In Silico Biol 1999, I(4): I 75- I82.
8. Anantharaman V, Koonin EV, Aravind L: Comparative genomics and evolution of proteins involved in RNA metabolism. Nucleic Acids Res 2002, 30(7): |427-|464.

9. Anantharaman V, Koonin EV, Aravind L: SPOUT: a class of methyltransferases that includes spoU and trmD RNA methylase superfamilies, and novel superfamilies of predicted prokaryotic RNA methylases. I Mol Microbiol Biotechnol 2002, 4(I):71-75

10. Schubert HL, Blumenthal RM, Cheng $X$ : Many paths to methyltransfer: a chronicle of convergence. Trends Biochem Sci 2003 , 28(6):329-335

II. Caldas T, Binet E, Bouloc P, Costa A, Desgres J, Richarme G: The Fts]/RrmJ heat shock protein of Escherichia coli is a 23 Sribosomal RNA methyltransferase. J Biol Chem 2000, 275(22): 164|4-164|9.

12. Bugl H, Fauman EB, Staker BL, Zheng F, Kushner SR, Saper MA, Bardwell JC, Jakob U: RNA methylation under heat shockcontrol. Mol Cell 2000, 6(2):349-360.

13. Persson BC, Jager G, Gustafsson C: The spoU gene of Escherichia coli, the fourth gene of the spoT operon, is essential for tRNA (Gm I 8) 2'-O-methyltransferase activity. Nucleic Acids Res 1997, 25(20):4093-4097.

14. Nureki O, Watanabe K, Fukai S, Ishii R, Endo Y, Hori H, Yokoyama S: Deep knot structure for construction of active site and cofactor binding site of tRNA modification enzyme. Structure (Camb) 2004, I 2(4):593-602.

15. Pintard L, Lecointe F, Bujnicki JM, Bonnerot C, Grosjean H, Lapeyre $B$ : Trm7p catalyses the formation of two 2'-O-methylriboses in yeast tRNA anticodon loop. Embo J 2002, 2 I (7): |8| |-|820.

16. Feder M, Pas J, Wyrwicz LS, Bujnicki JM: Molecular phylogenetics of the RrmJ/fibrillarin superfamily of ribose 2'-O-methyltransferases. Gene 2003, 302(I-2):129-138.

17. Dunin-Horkawicz S, Czerwoniec A, Gajda MJ, Feder M, Grosjean H, Bujnicki JM: MODOMICS: a database of RNA modification pathways. Nucleic Acids Res 2006, 34(Database):DI45-I49. 


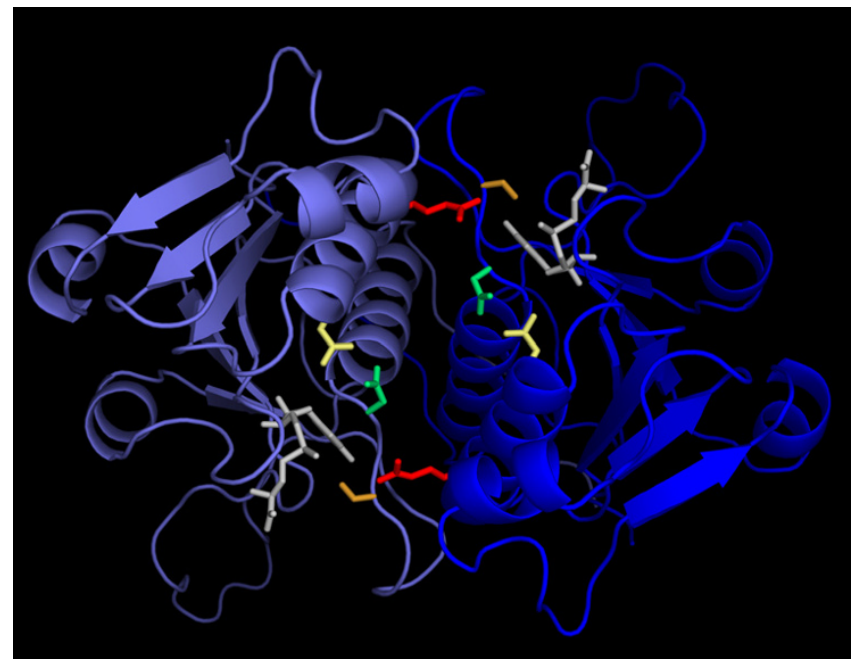

Figure I I

Three-dimensional model of the YfhQ dimer in complex with AdoMet. Both monomers are shown in the cartoon representation, in different shades of blue. AdoMet molecules (white) and residues predicted to be involved in catalysis in YfhQ and conserved in other ribose MTases (see Figure 7) are shown in the wireframe representation in different colors (NI7 in yellow, R23 in red, SI42 in orange, and $\mathrm{NI} 44$ in green). The coordinates of the model are available from the corresponding author upon request.

18. Gustafsson C, Reid R, Greene PJ, Santi DV: Identification of new RNA modifying enzymes by iterative genome search using known modifying enzymes as probes. Nucleic Acids Res 1996, 24(19):3756-3762.

19. Michel G, Sauve V, Larocque R, Li Y, Matte A, Cygler M: The structure of the RImB 23S rRNA methyltransferase reveals a new methyltransferase fold with a unique knot. Structure (Camb) 2002, 10:1303-1315.

20. Nureki O, Shirouzu M, Hashimoto K, Ishitani R, Terada T, Tamakoshi M, Oshima T, Chijimatsu M, Takio K, Vassylyev DG, et al: An enzyme with a deep trefoil knot for the active-site architecture. Acta Crystallogr D Biol Crystallogr 2002, 58(Pt 7): I I29-I I 37.

21. Saka K, Tadenuma M, Nakade S, Tanaka N, Sugawara H, Nishikawa K, Ichiyoshi N, Kitagawa M, Mori H, Ogasawara N, et al.: A complete set of Escherichia coli open reading frames in mobile plasmids facilitating genetic studies. DNA Res 2005, I 2(I):63-68.

22. Ito M, Baba T, Mori H: Functional analysis of I 440 Escherichia coli genes using the combination of knock-out library and phenotype microarrays. Metab Eng 2005, 7(4):3 I8-327.

23. Lim K, Zhang H, Tempczyk A, Krajewski W, Bonander N, Toedt J, Howard A, Eisenstein E, Herzberg O: Structure of the YibK methyltransferase from Haemophilus influenzae (HI0766): a cofactor bound at a site formed by a knot. Proteins 2003, $5 I(I): 56-67$.

24. Kurowski MA, Bujnicki JM: GeneSilico protein structure prediction meta-server. Nucleic Acids Res 2003, 3 I ( I 3):3305-3307.

25. Mosbacher TG, Bechthold A, Schulz GE: Structure and function of the antibiotic resistance-mediating methyltransferase AviRb from Streptomyces viridochromogenes. J Mol Biol 2005, 345(3):535-545.

26. Kosinski J, Cymerman IA, Feder M, Kurowski MA, Sasin JM, Bujnicki JM: A "FRankenstein's monster" approach to comparative modeling: merging the finest fragments of Fold-Recognition models and iterative model refinement aided by 3D structure evaluation. Proteins 2003, 53(Suppl 6):369-379.

27. Kosinski J, Gajda MJ, Cymerman IA, Kurowski MA, Pawlowski M, Boniecki M, Obarska A, Papaj G, Sroczynska-Obuchowicz P, Tkaczuk

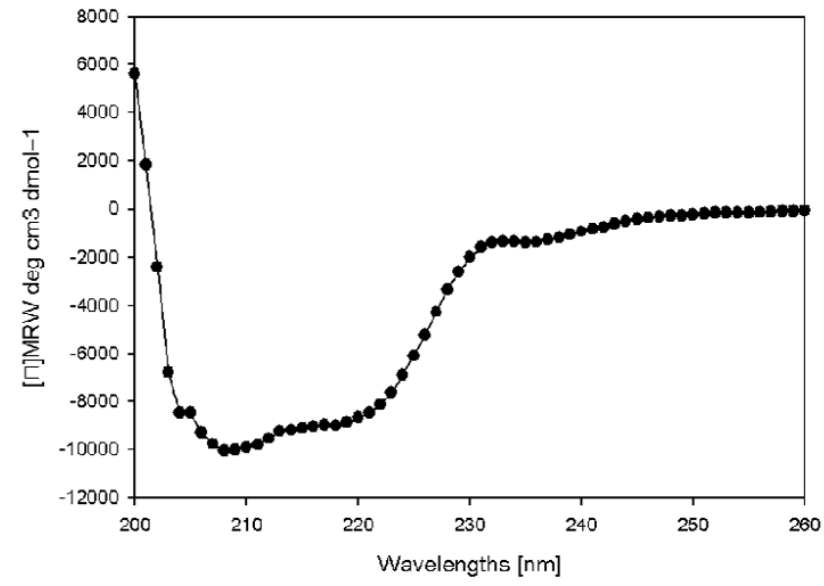

Figure 12

Far UV CD spectrum of YfhQ protein. Spectra were measured in $0.15 \mathrm{M}$ Tris- $\mathrm{HCl} \mathrm{pH} 7.5$ buffer and corrected by substraction of a buffer spectrum. The concentration of YfhQ protein was $6.6 \mu \mathrm{M}$. Three accumulations were measured, which were recorded in millidegrees every I $\mathrm{nm}$. The mean residue ellipticities for the spectra were calculated to aid in secondary structure determination by comparison with standard spectra for secondary structure elements.

$\mathrm{KL}$, et al:: FRankenstein becomes a cyborg: theautomatic recombination and realignment of Fold-Recognition models inCASP6. Proteins 2005.

28. Watanabe K, Nureki O, Fukai S, Ishii R, Okamoto H, Yokoyama S, Endo $Y$, Hori $H$ : Roles of conserved amino acid sequence motifsin the SpoU (TrmH) RNA methyltransferase family. J Biol Chem 2005, 280(I I): 10368-10377.

29. Lapeyre B: Conserved ribosomal RNA modification and their putative roles in ribosome biogenesis and translation. In Finetuning of RNA functions by modification and editing Volume 12. Edited by: Grosjean H. Berlin-Heidelberg: Springer-Verlag; 2005.

30. Altschul SF, Madden TL, Schaffer AA, Zhang J, Zhang Z, Miller W, Lipman DJ: Gapped BLAST and PSI-BLAST: a new generation ofprotein database search programs. Nucleic Acids Res 1997, 25( I 7):3389-3402.

31. Bjork GR, Jacobsson K, Nilsson K, Johansson MJ, Bystrom AS, Persson OP: A primordial tRNA modification required for the evolution of life? Embo / 200I, 20(I-2):23 I-239.

32. Christian T, Evilia C, Williams S, Hou YM: Distinct origins of tRNA(mIG37) methyltransferase. J Mol Biol 2004, 339(4):707-719.

33. Cavaille J, Chetouani F, Bachellerie JP: The yeast Saccharomyces cerevisiae YDLI I 2 w ORF encodes the putative 2'-O-ribose methyltransferase catalyzing the formation of $\mathrm{GmI8}$ in tRNAs. Rna 1999, 5(I):66-8I.

34. Bujnicki JM: In silico analysis of the tRNA:m' A58 methyltransferase family: homology-based fold prediction and identification of new members from Eubacteria and Archaea. FEBS Lett 200I, 507(2): I23-127.

35. Roovers M, Wouters J, Bujnicki JM, Tricot C, Stalon V, Grosjean H, Droogmans L: A primordial RNA modification enzyme: the case of tRNA ( $\left.\mathbf{m}^{1} \mathbf{A}\right)$ methyltransferase. Nucleic Acids Res 2004, 32(2):465-476.

36. Arnez JG, Steitz TA: Crystal structure of unmodifiedtRNA(GIn) complexed with glutaminyl-tRNA synthetase and ATP suggests a possible role for pseudo-uridines in stabilization of RNA structure. Biochemistry 1994, 33(24):7560-7567. 
37. Sreerama N, Woody RW: Estimation of protein secondary structure from circular dichroism spectra: comparison of CONTIN, SELCON, and CDSSTR methods with an expanded reference set. Anal Biochem 2000, 287(2):252-260.

38. Grosjean H, Keith G, Droogmans L: Detection and quantification of modified nucleotides in RNA using thin-layer chromatography. Methods Mol Biol 2004, 265:357-39l.

Publish with Bio Med Central and every scientist can read your work free of charge

"BioMed Central will be the most significant development for disseminating the results of biomedical research in our lifetime. " Sir Paul Nurse, Cancer Research UK

Your research papers will be:

- available free of charge to the entire biomedical community

- peer reviewed and published immediately upon acceptance

- cited in PubMed and archived on PubMed Central

- yours - you keep the copyright 